\title{
Propagación vegetativa de Myrceugenia exsucca y Blepharocalyx cruckshanksii, especies dominantes del bosque pantanoso de la Depresión Intermedia de la región de La Araucanía, Chile
}

\author{
Vegetative propagation of Myrceugenia exsucca and Blepharocalyx cruckshanksii, \\ swamp forest dominant species of the central depression of Araucanía region, Chile \\ ${\text { Mirtha Latsague Vidal }{ }^{a} \text {, Patricia Sáez Delgado }}^{\text {ab }}$, Enrique Hauenstein Barra ${ }^{a}$, Fernando Peña-Cortés ${ }^{a}$ \\ *Autor de correspondencia: aUniversidad Católica de Temuco, Escuela de Ciencias Ambientales, \\ Facultad de Recursos Naturales, casilla 15-D Temuco, Chile, tel.: (45)205409, fax: (45)211034, mlatsagu@uct.cl \\ bUniversidad de Concepción, Facultad de Ciencias Forestales, Concepción, Chile.
}

\begin{abstract}
SUMMARY
The Araucanía region in Chile is rich in wetlands, standing out the swampy forests of Myrtaceae located in the central depression, which are mentioned in the red book of the priority places for Chile's biological diversity conservation in the country. These forests are being intervened through drainage of their soils, logging and fret to fire, to obtain suitable soils for agriculture. The elimination of this type of ecosystems would mean an irreparable loss of the local biodiversity. The dominant species of this type of forest, Blepharocalyx cruckshanksii and Myrceugenia exsucca lack studies with regard to their regeneration capacity or vegetative propagation. The above mentioned situation can turn into a negative factor at the moment of planning strategies for their conservation. The aim of this work was to evaluate the capacity of in vivo rooting of semi hardwood stem cuttings of $B$. cruckshanksii and M. exsucca. The influence of exogenous auxins was analyzed. Cuttings collected in April 2008 at Pumalal sector, region of the Araucanía, Chile, were treated with indolbutyric acid (IBA) at different concentrations (0;1,000; 1,500; 2,000 and 2,500 mg L-1). After keeping the cuttings for four months in rooting beds with bottom heat, the survival percentage, stem rooting percentage and potential of root growth were evaluated. IBA only influenced rooting percentage and root length of B. cruckshanksii; with $41 \%$ and $4.7 \mathrm{~cm}$ in average, respectively in $2,000 \mathrm{mg} \mathrm{L}^{-1}$ concentration. In $M$. exsucca influence of IBA was not observed respect to control $(P>0.05)$.
\end{abstract}

Key words: Myrtaceae, cutting, stem rooting, IBA, wetland.

\section{RESUMEN}

La región de La Araucanía es rica en humedales, destacando los bosques pantanosos de mirtáceas ubicados en la Depresión Intermedia y mencionados en el libro rojo de los sitios prioritarios para la conservación de la diversidad biológica de Chile. Estos bosques están siendo intervenidos a través del drenaje de sus suelos, tala y roce a fuego con el objeto de obtener suelos aptos para la agricultura. La eliminación de estos ecosistemas significaría una pérdida irreparable de la biodiversidad del lugar. Las especies dominantes de estos bosques carecen de estudios respecto a sus capacidades de regeneración o reproducción vegetativa, situación que puede convertirse en un factor negativo a la hora de realizar propuestas o estrategias para su conservación. El objetivo fue evaluar la capacidad de enraizamiento in vivo de estacas semileñosas de B. cruckshanksii y M. exsucca probando distintas concentraciones de ácido indolbutírico (AIB) y su efecto sobre el desarrollo del sistema radicular. Estacas colectadas en abril del 2008 en el sector Pumalal, región de La Araucanía, fueron tratadas con AIB en distintas concentraciones (0, 1.000, $1.500,2.000$ y 2.500 $\mathrm{mg} \mathrm{L}^{-1}$ ). Después de mantener las estacas durante cuatro meses en cama caliente, se evaluaron los porcentajes de sobrevivencia y de enraizamiento y el potencial de crecimiento radicular. El AIB $\left(2.000 \mathrm{mg} \mathrm{L}^{-1}\right)$ sólo influyó en el porcentaje de enraizamiento y la longitud de raíces de B. cruckshanksii, con un promedio de $41 \%$ y $4,7 \mathrm{~cm}$, respectivamente; en $M$. exsucca no se observó su influencia $(P>0,05)$.

Palabras clave: Myrtaceae, estacas, rizogénesis, AIB, humedales.

\section{INTRODUCCIÓN}

La actividad humana es responsable de muchos tipos de degradación ambiental y, en particular, las masas forestales nativas están siendo amenazadas de agotamiento genético o de extinción, incluso antes de que sus características y variación genética sean conocidas (Abedini 2005). Ello ha hecho necesario enfocar las investigaciones a aquellas especies que cumplen otras funciones como, por ejemplo, el soporte de ecosistemas (Aparicio et al. 2009) que contribuyen a la protección de riberas, regulación del cauce de los ríos y retención de la sedimentación de las aguas. Por 
otro lado, se favorece también la nidificación y refugio de especies de fauna silvestre tales como Patagioenas araucana (Lesson) (torcaza) y Lontra provocax (Thomas) (huillín). En la región de La Araucanía, el bosque BlepharocalyoMyrceugenietum exsuccae corresponde a una asociación boscosa pantanosa, perennifolia, monoestratificada de unos $18 \mathrm{~m}$ de altura (Ramírez et al. 1995). Se encuentra en diferentes estados de degradación, siendo especialmente afectado por actividades de extracción de leña, tala y roce a fuego, junto a políticas gubernamentales de incentivos a la "recuperación de terrenos para la agricultura" (Hauenstein et al. 2002). La regeneración de estas áreas degradadas requiere que especialmente sus especies dominantes sean repobladas. Blepharocalyx cruckshanksii (Hook. et Arn.) Nied (temo) y Myrceugenia exsucca (D. C.) Berg (pitra) son especies de la familia Myrtaceae características de los bosques de humedal. Blepharocalyx cruckshanksii es una especie endémica de Chile, no muy frecuente; crece entre Colchagua (región de Valparaíso) y Chiloé (región de Los Lagos), a orillas de cursos de agua o en laderas sombrías y húmedas de exposición sur, desde el nivel del mar hasta los $400 \mathrm{~m}$ s.n.m. Tolera suelos de $\mathrm{pH}$ neutro a ácido y florece desde fines de diciembre hasta fines de febrero; los frutos maduran en marzo (González et al. 2003). Myrceugenia exsucca es una especie nativa de Chile y Argentina. Se encuentra desde la provincia de Choapa hasta Chiloé. Es un árbol higrófilo que crece sólo en lugares pantanosos o terrenos muy húmedos, junto a ríos, esteros y lagos y a menudo dentro del agua. Florece desde enero a mayo y fructifica de junio a septiembre (Donoso y Cabello 1978).

Hechenleitner et al. (2005) señalan la importancia de desarrollar estrategias efectivas destinadas a salvaguardar el futuro de algunas especies de plantas amenazadas del centro y sur de Chile, mediante estudio de sus sistemas de reproducción tanto sexual como vegetativa. Existe información respecto a la reproducción por semillas en géneros de la familia Myrtaceae. Así, se ha informado la influencia del almacenamiento por períodos variables en la viabilidad de semillas de Amomyrtus luma (Molina) Legrand et Kausel, Luma apiculata (D. C.) Burret, Myrceugenia planipes (Hook. et Arn.) Berg y Ugni molinae (Turcz) (Ramírez et al. 1980) y la importancia de los tratamientos pregerminativos, específicamente extracción de los frutos y siembra de semillas recién colectadas de Myrceugenia exsucca (Donoso 2006). Por su parte, Meza y Bautista (2007) evaluaron el efecto del remojo en agua en la germinación de semillas de Psidium guajava L. y Otegui et al. (2007) evaluaron el efecto de la temperatura y la luz en la germinación de semillas de Paspalum guenoarum Arechav.

Para la propagación vegetativa, cuando se quiere inducir raíces adventicias debe considerarse que la aptitud para formar nuevos individuos depende en gran medida de la especie y de las características del material vegetativo, como la consistencia del tejido, el ambiente de propagación, las técnicas y los tratamientos hormonales entre otros factores (Hartmann et al. 2002). Se sabe bien que las auxinas juegan un rol significativo en estimular el enraizamiento adventicio en estacas de tallo de especies leñosas (Kesari et al. 2009) y que las divisiones de las primeras raíces iniciadas son dependientes de los niveles exógenos y endógenos de auxinas (Ludwig-Müller 2000, Kochlar et al. 2005). A pesar de que existe información sobre reproducción asexual en especies de la familia Myrtaceae (Legrandia concinna (Philippi) Kausel, Myrceugenia colchaguensis (Phil.) Navas, Myrceugenia leptospermoides (D. C.) Kausel, Myrceugenia pinifolia (Phil.) Kausel, Myrceugenia rufa (Colla) Skottsb ex Kausel, Myrcianthes coquimbensis (Barneoud) Landrum et Grifo) (Hechenleitner et al. 2005), B. cruckshanksii y M. exsucca, las especies seleccionadas para este trabajo carecen de suficiente información respecto de su respuesta a las técnicas de reproducción asexual. Al respecto, Donoso (2006) señala la necesidad de investigar en torno al enraizamiento de estacas en estas especies, dada la potencialidad ornamental que poseen principalmente en bordes de canales o sectores con mucha humedad en el suelo.

El objetivo de este trabajo es evaluar la capacidad de enraizamiento in vivo de estacas semileñosas de B. cruckshanksii y $M$. exsucca aplicando distintas concentraciones de ácido indolbutírico y su efecto sobre el desarrollo del sistema radicular. Estos antecedentes permitirán aportar información básica para futuras investigaciones de programas de conservación de bosques de humedal.

\section{MÉTODO}

Se colectó material de 15 árboles adultos ubicados en el sector Pumalal (38 $36^{\circ}$ '34,80' S-72 $31^{\prime}$ ' 28,59', O y $38^{\circ} 35^{\prime}$ 55,20" S-72 31' 2,76" O) de la Depresión Intermedia, en la región de La Araucanía. La recolección del material vegetal se realizó durante el mes de abril de 2008. Se seleccionaron al azar estacas semileñosas de la sección media de la copa de individuos de aproximadamente 50 años de edad que presentaban tallos con crecimientos sanos, vigorosos y localizados en el borde del bosque. Después de la cosecha, el material vegetal fue inmediatamente trasladado al laboratorio en bolsas plásticas oscuras dentro de contenedores fríos. En el laboratorio se dimensionaron bajo agua estacas de $15 \mathrm{~cm}$ de longitud y $0,3 \mathrm{~cm}$ de diámetro, con presencia de al menos dos yemas visibles. Se dejaron como máximo un par de hojas en el tercio superior de la estaca, con un área foliar promedio de $0,08 \mathrm{dm}^{2}$. El medio de enraizamiento consistió en una mezcla de corteza compostada de Pinus radiata D. Don (G-15) de pH 5,5 y perlita, en una proporción 2:1. Se utilizaron almacigeras Aislapol $84 / 100^{\circledR}$. Las cavidades se llenaron en su totalidad con el sustrato indicado. 
Las concentraciones de ácido indolbutírico (AIB) utilizadas fueron $1.000 \mathrm{mg} \mathrm{L}^{-1}(\mathrm{~T} 1), 1.500 \mathrm{mg} \mathrm{L}^{-1}(\mathrm{~T} 2), 2.000 \mathrm{mg}$ $\mathrm{L}^{-1}$ (T3) y $2.500 \mathrm{mg} \mathrm{L}^{-1}$ (T4), más un tratamiento control (T0), se estandarizó el pH de las soluciones a 5,8. El AIB se disolvió en etanol absoluto grado reactivo (Merck). A las estacas se les realizaron cortes basales oblicuos en un recipiente con agua para evitar cavitación xilemática, sumergiendo luego durante $15 \mathrm{~min}$ en las soluciones hormonales correspondientes. La desinfección se realizó mediante la inmersión de las estacas en una solución con fungicida Captan $^{\circledR}$ en concentración $10 \mathrm{~g} \mathrm{~L}^{-1}$ durante 15 minutos previo al tratamiento auxínico. Las bandejas se mantuvieron en invernadero en una cama caliente a una temperatura constante de $25^{\circ} \mathrm{C}$ durante cuatro meses. La humedad relativa fue de $60 \% \pm 5 \%$ y la temperatura ambiental fluctuó entre 15 y $20^{\circ} \mathrm{C}$. Este rango se controló mediante una estufa termorregulada y un sistema de riego automatizado. Se aplicó riego tres veces al día durante un minuto, a través de un sistema de aspersores (microjet).

Se realizaron 10 réplicas por tratamiento con cinco estacas por réplica, utilizando un diseño completo aleatorio. Se descartaron las estacas muertas y en las restantes se tomaron los datos de enraizamiento, número y longitud de raíces. El efecto de la concentración de ácido indolbutírico en las variables sobrevivencia (\%) y enraizamiento (\%) se determinó con la prueba de Chi-cuadrado $(P=0,05)$ (Zar 1996). Para las variables número total de raíces y longitud de la raíz principal, se realizó un análisis de varianza de un factor $(P=0,05)$. Las diferencias significativas fueron determinadas mediante la prueba de rangos múltiples de Fisher (LSD).

\section{RESULTADOS}

Efectos del ácido indolbutírico en Blepharocalyx cruckshanksii. Luego de cuatro meses de permanencia de las estacas en invernadero, se formaron raíces en la zona proximal. Se observaron diferencias significativas entre los tratamientos, tanto para el porcentaje de supervivencia como para el de enraizamiento. Los mayores porcentajes de mortalidad se presentaron en estacas tratadas con las mayores concentraciones de ácido indolbutírico $(2.000 \mathrm{y}$ $2.500 \mathrm{mg} \mathrm{L}^{-1}$ ). Sin embargo, estas concentraciones promovieron el mayor porcentaje de enraizamiento $(41,1 \mathrm{y}$ $30,5 \%$, respectivamente) (cuadro 1).

Respecto al número de raíces, en $B$. cruckshanksii la aplicación de hormona no mostró tener influencia sobre esta variable $(P>0,05)$. En promedio presentaron 3,4 raíces por estaca. Por el contrario, la aplicación de auxina ácido indolbutírico estimuló la longitud de las raíces formadas $(P<0,05)$ (cuadro 1).

Efectos del ácido indolbutírico en Myrceugenia exsucca. Luego de cuatro meses de mantenidas las estacas en invernadero, la aplicación de auxina ácido indolbutírico

Cuadro 1. Efecto de la concentración de ácido indolbutírico (AIB) en la rizogénesis de estacas de B. cruckshanksii y de M. exsucca. Medias \pm desviación estándar. Letras distintas indican diferencias significativas entre tratamientos $(P<0,05)$.

Effect of IBA concentration on the rooting capacity of B. cruckshanksii and $M$. exsucca. Average \pm standard error. Different letters indicate significant differences $(P<0.05)$.

\begin{tabular}{|c|c|c|c|c|}
\hline \multirow{2}{*}{$\begin{array}{l}\text { Tratamiento } \\
\text { AIB }\left(\mathrm{mg} \mathrm{L}^{-1}\right)\end{array}$} & \multirow{2}{*}{$\begin{array}{l}\text { Supervivencia } \\
\text { (\%) }\end{array}$} & \multirow[b]{2}{*}{ Enraizamiento $(\%)$} & \multicolumn{2}{|c|}{ Desarrollo radicular } \\
\hline & & & $\begin{array}{l}\text { Número de raíces } \\
\text { (N\%/estaca) }\end{array}$ & $\begin{array}{l}\text { Longitud de raíces } \\
\qquad(\mathrm{cm})\end{array}$ \\
\hline & \multicolumn{4}{|c|}{ Blepharocalyx cruckshanksii } \\
\hline 0 & $45,5 \pm 15,7 \mathrm{bc}$ & $7,0 \pm 6,1 \mathrm{a}$ & $3,0 \pm 2,6 \mathrm{a}$ & $1,5 \pm 1,3 \mathrm{a}$ \\
\hline 1.000 & $51,5 \pm 5,2 \mathrm{c}$ & $15,9 \pm 16,7 \mathrm{ab}$ & $2,9 \pm 1,0 \mathrm{a}$ & $3,7 \pm 1,5 b$ \\
\hline 1.500 & $51,5 \pm 6,4 \mathrm{c}$ & $18,5 \pm 16,9 \mathrm{ab}$ & $3,4 \pm 1,7 \mathrm{a}$ & $4,2 \pm 1,5 \mathrm{~b}$ \\
\hline 2.000 & $21,2 \pm 5,3 \mathrm{a}$ & $41,1 \pm 8,4 \mathrm{c}$ & $3,7 \pm 1,6 \mathrm{a}$ & $4,7 \pm 1,7 \mathrm{~b}$ \\
\hline \multirow[t]{2}{*}{2.500} & $27,2 \pm 9,1 \mathrm{ab}$ & $30,5 \pm 4,7 \mathrm{bc}$ & $3,8 \pm 1,3$ a & $4,9 \pm 1,3 \mathrm{~b}$ \\
\hline & \multicolumn{4}{|c|}{ Myrceugenia exsucca } \\
\hline 0 & $81,8 \pm 9,1$ a & $10,3 \pm 0,58 \mathrm{a}$ & $3,7 \pm 0,6 \mathrm{a}$ & $3,3 \pm 0,49 \mathrm{ab}$ \\
\hline 1.000 & $78,7 \pm 10,5$ a & $11,4 \pm 10,3 \mathrm{a}$ & $3,5 \pm 1,3 \mathrm{a}$ & $4,3 \pm 0,67 \mathrm{~b}$ \\
\hline 1.500 & $63,6 \pm 15,1 \mathrm{a}$ & $13,0 \pm 11,5 \mathrm{a}$ & $2,9 \pm 1,4 \mathrm{a}$ & $2,9 \pm 1,44 \mathrm{a}$ \\
\hline 2.000 & $69,7 \pm 10,5$ a & $26,7 \pm 11,1 \mathrm{a}$ & $3,1 \pm 1,1 \mathrm{a}$ & $3,1 \pm 1,07 \mathrm{ab}$ \\
\hline 2.500 & $75,7 \pm 13,9$ a & $17,5 \pm 15,2$ a & $2,0 \pm 1,4 \mathrm{a}$ & $4,0 \pm 2,30 \mathrm{ab}$ \\
\hline
\end{tabular}


no influyó sobre el porcentaje de supervivencia y enraizamiento, no existiendo diferencias significativas entre el tratamiento control y los demás tratamientos, lo que arrojó en promedio, 73,9 y 15,8\% de supervivencia y enraizamiento, respectivamente (cuadro 1).

Al igual que en las variables supervivencia y enraizamiento, la aplicación de distintas concentraciones de auxina en $M$. exsucca no produjo diferencias significativas $(P>0,05)$ en las variables asociadas al desarrollo radicular. A pesar de existir una leve disminución en el número de raíces con el aumento en la concentración hormonal, ésta no difirió estadísticamente entre tratamientos, encontrándose en promedio 3,1 raíces por estaca con una longitud media de $3,4 \mathrm{~cm}$ (cuadro 1).

\section{DISCUSIÓN}

Son variados los factores que condicionan el éxito en el proceso de enraizamiento. En general, los porcentajes de enraizamiento obtenidos en este ensayo son bajos comparados con otras especies nativas investigadas, tales como Desfontainia spinosa (Ruiz et Pav.) y Laureliopsis philippiana (Looser) Schodde (Delgado et al. 2008), lo que podría estar relacionado con el tipo de estaca, época de recolección y concentraciones hormonales utilizadas. Los bajos porcentajes de supervivencia y enraizamiento obtenidos se pueden atribuir a que la mayoría de las estacas perdió sus hojas durante el ensayo. Como consecuencia de la pérdida de las hojas, la tasa fotosintética decae más rápidamente que la tasa respiratoria, así se induce la expresión del gen de la gliceraldehído fosfato deshidrogenasa citosólica activándose la glicólisis, el resultado final en este caso es la reducción de las reservas de carbohidratos cofactores del enraizamiento (Azcon Bieto y Talón 2000). Las estacas tratadas fueron recolectadas en época de floración y maduración de los frutos, procesos que se caracterizan por cambios en la distribución de los asimilados y nutrientes (Barceló et al. 1995). Un enraizamiento con estacas sin presencia de flores podría ser mejor para estos fines, lo que concuerda con Rodríguez et al. (2005), quienes señalan que la adquisición de la capacidad de florecer es un criterio consistente para asegurar el inicio del estado de madurez, y con ello el descenso de la capacidad para formar órganos adventicios. Según Hechenleitner et al. (2005), bajos porcentajes de enraizamiento ocurren cuando se presenta un estrés hídrico. En este caso, es posible que la frecuencia de riego o la humedad ambiental no fuesen las adecuadas. Los resultados sugieren la necesidad de mantener una alta humedad relativa alrededor de las estacas durante la propagación para minimizar la pérdida de agua (Henselova 2002). El mayor porcentaje de supervivencia de las estacas de $M$. exsucca responde a su menor sensibilidad a las condiciones de humedad del ambiente, lo cual sería atribuible a la autoecología de la especie. Myrceugenia exsucca posee un rango de distribución mayor que
B. cruckshanksii (González et al. 2002). Según Gallo et al. (2004), la amplitud del rango de distribución influye en la plasticidad de las especies. Adicionalmente, $M$. exsucca presenta una madera liviana con gran cantidad de espacios o poros, lo que le permite una mayor oxigenación desde el tallo hacia las raíces (Ramírez et al. 1995).

La falta de asociación significativa entre el enraizamiento y la aplicación de ácido indolbutírico en $M$. exsucca concuerda con los resultados obtenidos para Berberidopsis corallina Hook.f. por Latsague et al. (2008). Sin embargo, los bajos porcentajes obtenidos en este ensayo sugieren evaluar la aplicación de mayores dosis hormonales. Por el contrario, B. cruckshanksii es más sensible a la aplicación de ácido indolbutírico por cuanto se observa un aumento significativo del enraizamiento en concentraciones superiores a $1.500 \mathrm{mg} \mathrm{L}^{-1}$ de ácido indolbutírico.

Los resultados obtenidos en $B$. cruckshanksii respecto a la influencia del AIB en la longitud de las raíces formadas corroboran lo informado por Mesén et al. (1997) y Palanisamy et al. (1998), quienes señalan que la aplicación de auxina mejora el desarrollo de las raíces adventicias. Sin embargo, el número de raíces formadas en ambas especies estudiadas no fue influenciado por la aplicación de hormona, a diferencia de lo informado por Abedini (2005), quien reporta diferencias significativas respecto al número de raíces en estacas de Parkinsonia aculeata L. tratadas con ácido indolbutírico.

En conclusión, de los parámetros evaluados, la hormona AIB sólo influyó en el porcentaje de enraizamiento y la longitud de raíces de B. cruckshanksii. En $M$. exsucca el ácido indolbutírico no mejoró los resultados con respecto al control. Según esto, resultaría interesante probar otras concentraciones o interacciones hormonales, como asimismo, distintos grados de lignificación de las estacas, con la finalidad de mejorar estos porcentajes y poder definir una metodología de reproducción vegetativa adecuada para estas especies.

\section{AGRADECIMIENTOS}

Los autores agradecen a la Dirección General de Investigación y Postgrado de la Universidad Católica de Temuco, a través del proyecto DGIP-UCT 2008-3-01.

\section{REFERENCIAS}

Azcon Bieto J, M Talón. 2000. Fundamentos de fisiología vegetal. Barcelona, España. Editorial McGraw-Hill Interamericana. $520 \mathrm{p}$.

Abedini W. 2005. Propagación vegetativa de Parkinsonia aculeata L. por estaquillado. Quebracho 12:23-33.

Aparicio A, M Pastorino, A Martines-Meir, L Gallo. 2009. Vegetative propagation of patagonian cypress, a vulnerable species from the subantartic forest of South America. Bosque 30(1): 18-26. 
Barceló Coll J, G Nicolás, B Sabater, R Sánchez. 1995. Fisiología vegetal. Madrid, España. Ediciones Pirámide. 662 p.

Delgado M, M Cuba, P Hechenleitner, O Thiers. 2008. Propagación vegetativa de taique (Desfontainia spinosa) y tepa (Laureliopsis philippiana) con fines ornamentales. Bosque 29(2): 120-126.

Donoso C. 2006. Especies arbóreas de los bosques templados de Chile y Argentina. Autoecología. Valdivia, Chile. Marisa Cúneo Ediciones. 678 p.

Donoso C, A Cabello. 1978. Antecedentes fenológicos y de germinación de las especies leñosas chilenas. Ciencias Forestales 1 (2): 31-41.

Gallo LA, MJ Pastorino, C Donoso. 2004. Variación en Austrocedrus chilensis (D. Don) Pic. Ser. et Bizzarri (ciprés de la cordillera). In Donoso C, A Premoli, L Gallo, R Ipinza eds. Variación intraespecífica en las especies arbóreas de los bosques templados de Chile y Argentina. Santiago, Chile. Editorial Universitaria. p. 233-251.

González M, E Hauenstein, F Peña-Cortés, M García, O Urrutia. 2003. Comentarios sobre bosques pantanosos, humedales importantes del centro-sur de Chile. Gestión Ambiental 9: 3-13.

Hartmann HT, DE Kester, FT Davies Jr, RL Geneve. 2002. Plant propagation: Principles and practices. New Jersey, USA. Prentice Hall. 880 p.

Hauenstein E, M González, F Peña, A Muñoz. 2002. Clasificación y caracterización de la flora y vegetación de los humedales de la costa de Toltén (IX Región de Chile). Gayana Botánica 59(2): 87-100.

Hechenleitner P, M Gardner, P Thomas, C Echeverría, B Escobar, P Brownless, E Martínez. 2005. Plantas amenazadas del Centro Sur de Chile. Distribución, conservación y propagación. Valdivia, Chile. Universidad Austral de Chile-Real Jardín Botánico de Edimburgo. 188 p.

Henselova M. 2002. Synergistic effect of benzolinone with IBA and fungicides on the vegetative propagation of ornamental plants, park and fruit woody species. J. Hortic Sci. 29: 41-50.

Kesari V, A Krishnamachari, L Rangan. 2009. Effect of auxins on adventitious rooting from stem cuttings of candidate plus tree Pongamia pinnata (L.), a potential biodisel plant. Trees 23: 597-604.
Kochhar VK, SP Singh, RS Katiyar, P Pusapangadan. 2005. Differential rooting and sprouting behaviour of two Jatropha species and associated physiological and biochemical changes. Curr. Sci. 89(6): 936-939.

Latsague M, P Sáez, E Hauenstein. 2008. Inducción de enraizamiento en estacas de Berberidopsis corallina con ácido indolbutírico. Bosque 29(3): 227-230.

Ludwing-Mûller J. 2000. Indole-3-butyric acid in plant growth and development. Plant Growth Regul. 32: 219-230.

Mesén F, AC Newton, RR Leakey. 1997. Vegetative propagation of Cordia allidora (Ruiz and Pavon) Oken: the effect of IBA concentration, propagation medium and cutting origin. For. Ecol. Manage. 92: 45-54.

Meza N, D Bautista. 2007. Morfología de semillas de guayabo (Psidium guajava L.), germinación y emergencia después del remojo en agua. Rev. Fac. Agron. 24 (1): 265-270.

Palanisamy K, SA Ansari, K Pramod, BN Gupta. 1998. Adventitious rooting in shoot cutting of Azadirachta indica and Pongamia pinnata. New For. 16: 81-88.

Otegui M, MA Pérez, M de Souza Maia. 2007. Efecto de la temperatura y la luz en la germinación de semillas de Paspalum guenoarum. Revista Brasileira de Sementes 27(1): 190-194.

Ramírez C, M Romero, O Henríquez. 1980. Estudios de germinación en semillas de mirtáceas chilenas. Bosque 3(2): 106-114.

Ramírez C, C San Martín, J San Martín. 1995. Estructura florística de los bosques pantanosos de Chile Sur-Central; p. 215-234. In Armesto JJ, C Villagrán, M Kalin Arroyo eds. Ecología de los bosques nativos de Chile. Santiago, Chile. Universitaria. $470 \mathrm{p}$.

Rodríguez R, M Fernández, J Pacheco, MJ Cañal. 2005. Envejecimiento vegetal, una barrera a la propagación. Alternativas. In Ríos D, M Sánchez-Olate eds. Biotecnología vegetal en especies leñosas de interés forestal. Concepción, Chile. Facultad de Ciencias Forestales, Universidad de Concepción. $164 \mathrm{p}$.

Zar JH. 1996. Biostatistical analysis. London, UK. Prentice Hall International. $662 \mathrm{p}$.

Recibido: 21.04.10 Aceptado: 07.07.10 